\title{
Perfil sociodemográfico da população que demanda assistência médico-hospitalar em região do Estado de São Paulo, Brasil, 1988*
}

\author{
The social-demographic profile of the population seeking health services \\ in a region of the State of S. Paulo, Brazil, 1988
}

\author{
Rafael de Jesus Montero D'Oleo**, Manildo Fávero
}

\begin{abstract}
MONTERO D'OLEO, R. de J. \& FÁVERO, M. Perfil sociodemográfico da população que demanda assistência médico-hospitalar em região do Estado de São Paulo, Brasil, 1988. Rev. Saúde públ., S.Paulo, 26: 256-63, 1992. Fez-se um estudo descritivo, retrospectivo do Perfil Sócio-Econômico da População que demanda Assistência Médico-Hospitalar em uma Região do Nordeste do Estado de São Paulo (Brasil), comparando-se os resultados de pesquisas anteriores. Foram estudados 126.297 egressos de 25 hospitais gerais da Região em 1988, segundo sexo, grupo etário, local de residência, fonte de financiamento e coeficiente de internação. Os resultados mostraram que a distribuição da demanda segundo sexo e idade é semelhante à conhecida na literatura. Sugere-se que a regionalização da assistência, apesar dos planos e da legislação pertinente, é insatisfatória e fortemente influenciada pela presença de grandes centros urbanos e pelo desenvolvimento socioeconômico. Há participaçãa de várias modalidades de financiamento e diminuição da participação dos órgãos oficiais ao longo do tempo. A região estudada apresentou elevado coeficiente de internação aliado à baixa utilização de leitos hospitalares.
\end{abstract}

Descritores: Pesquisa sobre serviços de saúde. Hospitais gerais, estatisticas. Necessidades e demanda de serviços de saúde, tendências. Fatores sócio-econômicos.

\section{Introdução}

A instituição de prestação de serviço (centros de saúde, hospitais, unidades básicas de saúde e outros) é o meio por excelência para a explicitação das políticas de saúde, concretizadas através de planos e programas assistenciais. É nestas mesmas instituições que são expressas as contradições e as diferenças dos distintos grupos da sociedade, através do perfil de saúde-doença. Os serviços de saúde podem ser definidos como "o exercício legitimado da medicina, da odontologia e outras práticas de saúde pública, cujo fim explícito é preservar a saúde da população" 17 .

A análise ou avaliação da eficiência/efícácia dessas instituições pode ser um instrumen-

* Parte da Dissertação de Mestrado apresentada à Faculdade de Saúde Pública da USP, em 1991, subordinada ao titulo "Utilização dos leitos hospitalares na Região de Ribeirão Preto, 1988".

* Departamento de Prática de Saúde Pública da Faculdade de Saúde Pública da USP - São Paulo, SP - Brasil.

*** Departamento de Medicina Preventiva e Social da Faculdade de Ciências Médicas da Universidade de Campinas - Campinas, SP - Brasil.

Separatas/Reprints: R. de J.M.D'Oleo - Av. Dr. Amaldo, 715 - 01246-904 - São Paulo, SP - Brasil.

Publicação financiada pela FAPESP. Processo Saúde Coletiva 91/4994-0 to para medir a efetividade das políticas de saúde. Esta é uma das preocupações constantes quando de sua formulação; é assim que, em geral, os planos de saúde abordam a necessidade de melhora da eficiência, qualidade e cobertura dos serviços de saúde dando ênfase ao sistema hospitalar. O hospital é extremamente dispendioso, não só pela sua construção, mas principalmente pelo seu custeio, o que leva diferentes países, principalmente os subdesenvolvidos, a deficiências no número de leitos hospitalares necessários para garantir a recuperação da saúde, sobretudo das populações marginalizadas ou carentes. Esta situação vem se agravando nos últimos anos, visto o aumento desproporcionado dos custos e a falta de uma política coerente com as necessidades e o perfil epidemiológico da população.

No contexto atual, as instituições hospitalares caracterizam-se por oferecer assistência à saúde, de caráter eminentemente curativo, com uso extensivo de tecnologia de ponta e com custos cada vez mais elevados. O hospital como instituição indispensável para a recuperação da saúde e prevenção da doença é cenário apropriado para a análise do uso dos recursos dentro do sistema de prestação de serviços de saúde. Esta análise poderá ser feita 
utilizando diversos tipos de estudos, a saber: de morbidade, de morbidade hospitalar, de demanda, análise dos indicadores de uso hospitalar, além de outras informações.

Grande número de recomendações relativas à necessidade de leitos/1.000 habitantes tem surgido, muitas vezes até sem justificativas, ou baseadas nos mais diversos raciocinios. $O$ índice mais aceito e que os autores do setor saúde repetem ou citam constantemente é 4,5 leitos $/ 1.000$ hapitantes, recomendado pela Organização Panamericana da Saúde para América Latina ${ }^{12,13}$.

Embora sejam conhecidas e difundidas várias abordagens metodológicas para conhecer as necessidades de leitos hospitalares ${ }^{10}$, elas são pouco utilizadas e novos hospitais vão sendo construídos, ou reivindicados por grupos populacionais e até por profissionais de saúde sem atender ao planejamento da rede hospitalar, provocando excesso de leitos, situação esta que se agrava pelo desperdício no uso dos leitos já existentes.

Este desperdício pode ocorrer por duas maneiras. Primeiro por subutilização, que ocorre quando o hospital não chega a utilizar seus leitos em um nível aceitável, em outras palavras, trabalha com taxas de ocupação abaixo do recomendado. Segundo por utilização inadequada, que ocorre em hospitais com elevada taxa de ocupação, porém com duraçảo da internação aumentada ${ }^{6}$.

A necessidade de se conhecer o perfil sociodemográfico da população que demanda algum tipo de assistência à saúde é cada vez mais importante, tendo em vista que a utilização dos serviços de saúde é produto de um conjunto de interações entre os profissionais da saúde e seus clientes, ocorrendo esta dentro de um ambiente organizacional que, por sua vez, é rodeado e permeado por traços sociais e culturais ${ }^{3}$. Embora esta grande variedade de fatores sejam discutidos como entidades independentes, deve-se ter em mente que elas geralmente são indissociáveis. Assim, uma investigação epidemiológica descritiva com fins administrativos, de planejamento ou de política de saúde, deverá descrever a utilização dos serviços de saúde especificamente para cada um dos atributos sociodemográficos (idade, sexo, estado civil, nivel socioeconômico, e outros) dos usuários dos serviços. "A epidemiogia analítica trata dos fatores sociodemográficos como determinantes do uso dos serviços de saúde. Para determinado grau de doença, será que alguns grupos de idade, sexo, raça, estado civil ou socioeconômico tenderiam a fazer um maior uso dos serviços de saúde, presumindo-se a mesma acessibilidade geográfica e econômica? Infelizmente, pouca pesquisa tem sido feita sobre este tópico" ${ }^{4}$.

O objetivo do presente trabalho é conhecer as características sociodemográficas dos pacientes que utilizaram os leitos hospitalares da Região de Ribeirão Preto, bem como o coeficiente de internação da região referida.

\section{Material e Método}

Foi feito estudo retrospectivo da ultilização dos leitos hospitalares da região administrativa de saúde de Ribeirão Preto, localizada no extremo nordeste do Estado de São Paulo (Brasil). É uma das mais ricas regiōes do Estado de São Paulo, destacando-se o Município de Ribeirão Preto como centro de ensino médico com grande quantidade de recursos para a saúde.

O universo de estudo foi constituído dos pacientes egressos (altas e óbitos) ocorridos no ano de 1988 , de 25 hospitais que estão integrados ao sistema de processamento de dados hospitalares do Departamento de Medicina Social da Faculdade de Medicina de Ribeirão Preto da Universidade de São Paulo. As informaçōes dos pacientes foram extraídas da folha de alta hospitalar, instrumento utilizado para medir as seguintes variáveis: idade, sexo e procedência dos pacientes, além das fontes de financiamento das internações. Cada uma destas variáveis foi analisada segundo as definições estabelecidas pelas normas do Centro de Processamento de Dados Hospitalares (CPDH) 5 .

Durante o ano de 1988 o CPDH recebeu 146.505 folhas de alta hospitalar, representando os egressos que ocorreram em 28 dos 29 hospitais que formam parte da rede hospitalar.

Excluídos os egressos de recém-nascidos e os egressos de três hospitais psiquiátricos restaram 126.297 folhas de alta, que formaram o universo estudado e a partir das quais foram processadas as informações.

\section{Resultados e Discussão}

Dos 126.297 egressos estudados, 40,805 eram de pacientes do sexo masculino e 59.205 do feminino, se computados os casos corres- 
Tabela 1. Distribuição dos egressos hospitalares segundo grupo etário e sexo. Região de Ribeirão Preto, 1988.

\begin{tabular}{crrrrrr}
\hline $\begin{array}{c}\text { Grupo Etário } \\
\text { (anos) }\end{array}$ & \multicolumn{7}{c}{ Masculino } & \multicolumn{3}{c}{ Sexo } & Total \\
\cline { 2 - 7 } * & $\mathrm{N}$ & $\%$ & $\mathrm{~N}$ & $\%$ & $\mathrm{~N}$ & $\%$ \\
1 de 1 & 4.738 & 9,2 & 3.574 & 4,8 & 8.312 & 6,6 \\
5 a 9 & 3.481 & 6,8 & 2.842 & 3,8 & 6.323 & 5,0 \\
10 a 14 & 5.161 & 10,0 & 3.826 & 5,1 & 8.987 & 7,1 \\
15 a 19 & 2.104 & 4,1 & 1.567 & 2,1 & 3.670 & 2,9 \\
20 a 24 & 2.695 & 5,2 & 6.734 & 9,0 & 9.429 & 7,5 \\
25 a 29 & 2.939 & 5,7 & 10.384 & 13,9 & 13.323 & 10,5 \\
30 a 34 & 3.135 & 6,1 & 10.164 & 13,6 & 13.299 & 10,5 \\
35 a 39 & 3.048 & 5,9 & 7.413 & 9,9 & 10.461 & 8,3 \\
40 a 49 & 2.671 & 5,2 & 4.898 & 6,5 & 7.569 & 6,0 \\
50 a 59 & 5.207 & 10,1 & 6.604 & 8,8 & 11.811 & 9,4 \\
60 a 69 & 5.700 & 11,1 & 5.676 & 7,6 & 11.376 & 9,0 \\
70 ou mais & 5.066 & 9,8 & 4.807 & 6,4 & 9.873 & 7,8 \\
\hline Total & 5.588 & 10,8 & 6.271 & 8,4 & 11.856 & 9,4 \\
\hline
\end{tabular}

* Excluidos os recém-nascidos

(9) Excluídos 5 casos de sexo discriminado

pondentes às doenças da gravidez, parto $\mathrm{e}$ puerpério. Excluindo esses casos o sexo masculino passa para $51,3 \%$ e o feminino para $48,7 \%$, observando-se pequena predominância do sexo masculino, grupo este que também predomina na distribuição por sexo da população da sub-região.

$\mathrm{Na}$ Tabela 1 observa-se que os menores de um ano participaram com apenas $6,6 \%$; as maiores percentagens de internaçōes foram feitas em pacientes dos grupos estários de 20 a 24 anos e entre os de 25 a 29 anos $(10,5 \%)$. O grupo etário que contribuiu com a menor percentagem dos atendimentos foi $o$ de 10 a $14(2,9 \%)$. Pesquisa realizada no Vale do $\mathrm{Pa}$ raiba $^{8}$ constatou que as maiores percentagens de atendimentos ocorreram no grupo de 15 a 24 anos e nos menores de um ano.

Analisando-se os egressos por grupos etários e sexo, observa-se que o sexo masculino predominou percentualmente nos atendimentos nas faixas etárias de 0 a 14 anos e de 40 a 70 ou mais anos, perfazendo $30,0 \%$ e $41,8 \%$, respectivamente. Houve 25,923 diagnósticos atribuídos ao grupo XI da Classificação Internacional de Doenças - complicações da gravidez, do parto e do puerpério. O total de diagnóstico do sexo feminino correspondentes aos grupos etários de 15 a 49 anos dão o total de 46.197 casos.

Subtraindo-se deste total os 25.923 diagnósticos próprios do sexo feminino, teremos para este grupo etário 20.274 atendimentos. Este número é muito próximo dos 19.687 atendimentos encontrados no sexo masculino, do mesmo grupo etário. Parece razoável concluir que a maior demanda por assistência médico-hospitalar no grupo etário de 15 a 49 anos no sexo feminino se deve a complicaçōes da gravidez, parto e puerpério, ou outras causas inerentes à patologia feminina. Tendência semelhante foi encontrada nos Estados Unidos, onde aproximadamente $40 \%$ dos atendimentos ao sexo feminino dessa faixa de idade foi por este diagnóstico ${ }^{16}$.

Ao serem calculados os coeficientes de internações por idade (Tabela 2), observa-se que o grupo de 70 ou mais anos teve o mais alto coeficiente de internação, 472,69, fato aliás, esperado, pois sabe-se que os idosos apresentam alto coeficiente de morbidade hospitalar ${ }^{1,8,9,18}$. O grupo etário de 60 a 69 anos também apresentou elevado coeficiente de 267,1. Este comportamento da demanda pela população idosa, também foi observado nos Estados Unidos, embora o coeficiente de internação desta faixa etária, 65 a 74 anos, tenha apresentado uma queda nos últimos anos, passando de 324,3/1.000 habitantes em 1982 para 296,7 em 1986 e no grupo de 75 anos e mais caiu de 511,4 para 470,5 no mesmo período ${ }^{16}$. Dentre os demais grupos etários, os menores de um ano são os que mais apresentaram hospitalizações $(343,4)$, resultado semelhante aos 
Tabela 2. Coeficiente de internaçāo da Regiāo de Ribeirão Preto segundo grupo etário e sexo.

\begin{tabular}{cccc}
\hline $\begin{array}{c}\text { Grupo Etário } \\
\text { (anos) }\end{array}$ & Masculino & Sexo & ambos sexos \\
\cline { 2 - 4 } < de 1 & 381,4 & 303,4 & 343,4 \\
1 a 4 & 88,1 & 76,6 & 82,6 \\
5 a 9 & 113,6 & 88,5 & 101,3 \\
10 a 14 & 51,1 & 40,8 & 47,2 \\
15 a 19 & 68,3 & 180,1 & 122,8 \\
20 a 24 & 70,2 & 261,3 & 163,3 \\
25 a 29 & 77,0 & 261,8 & 167,3 \\
30 a 34 & 89,6 & 219,7 & 154,4 \\
35 a 39 & 96,8 & 177,4 & 136,3 \\
40 a 49 & 127,2 & 158,6 & 143,3 \\
50 a 59 & 187,6 & 180,9 & 182,7 \\
60 a 69 & 279,9 & 243,8 & 267,1 \\
70 ou mais & 505,8 & 448,5 & 472,7 \\
\hline Geral & 122,3 & 180,4 & 151,10 \\
\hline
\end{tabular}

encontrado por outros autores ${ }^{8,9,18}$. A faixa etária menos representada nos agressos hospitalares foi a de 10 a 14 anos, com 47,2 aproxidamente 7 vezes menor que o grupo de um ano.

O coeficiente geral de internações por mil habitantes foi de 151,1 , situando-se o sexo masculino abaixo desse valor $(122,3)$. Nos Estados Unidos o coeficiente em 1986 foi de 143,3 , o da região de Ribeirão Preto foi maior que o daquele país e da maioria dos países da América Latina e do Caribe ${ }^{15}$. Observa-se, também, que este coeficiente $(151,1)$ está acima daquele mencionado no Plano Decenal de Saúde para as Américas, para o período de 1971 a $1980^{14}$.

Embora o coeficiente de internação da região estudada seja de 151,10, quando se analisa o Município de Ribeirão Preto, separadamente dos demais municípios da região, observa-se imfortante diferença, ou seja, 168,3 e 133,4 respectivamente.

O coeficiente de internação no Município de Ribeirão Preto vem aumentando de 121,5 e 140,9 internações por 1.000 habitantes, entre 1972 e $1976^{19,20}$, e 168,3 , em 1988.

Newell ${ }^{11}$ afirma que a demanda pode ser induzida pela oferta de recursos e se for levado em consideração que a taxa de ocupação dos leitos hospitalares de Ribeirão Preto, em 1988, foi de apenas $57,6 \%$, parece lícito concluir que o excesso de leitos disponível aliado ao modelo privativista-mercantil dominante no pais, tenha induzido aumento da demanda de internações, apesar da política de restrição vigente nos últimos anos, implementada a partir do plano de reorientação da assistência a saúde no âmbito da Previdência Social, do Conselho Consultivo de Administração de Saúde Previdenciária. Nos grupos etários entre 15 e 49 anos o coeficiente de internação é maior no sexo feminino. $O$ sexo masculino apresentou os coeficientes mais elevados para os grupos etários extremos, isto é, de menos de um ano $(381,4)$, e de 70 ou mais anos $(505,8)$.

O coeficiente de internação por mil habitantes nos Estados Unidos em 1986, foi de 120,5 para o sexo masculino e de 164,3 para o sexo feminino ${ }^{7}$, e na Região de Ribeirão Preto, pouco mais elevados, 122,3 para masculino, e de 180,4 para feminino.

$O$ coeficiente de internação por mil habitantes não deve ser considerado como uma real medida do risco de internação da população e sim como uma relação numérica dos casos de hospitalizações por habitantes. $E$ isto porque, quando se calcula este coeficiente não está implícita a noção de risco de uma determinada população ser internada, pois não se conhece o número de pessoas verdadeiramente expostas a este risco.

A procedência é uma variável importante do ponto de vista administrativo, pois permite conhecer a movimentação dos pacientes dentro de regiōes e sub-regiōes, a procura de assistência hospitalar, sobretudo quando dentro do próprio sistema de saúde se dá ênfase ao uso dos recursos locais disponíveis. Esta variável também tem importância do ponto de vista epidemiológico.

$\mathrm{Na}$ Tabela 3 observa-se que dos 71.271 atendimentos feitos nos 9 hospitais do Muni- 
Tabela 3 - Número e percentagem de egressos hospitalares segundo procedência e local de atendimento.

\begin{tabular}{|c|c|c|c|c|c|c|}
\hline \multirow{3}{*}{ Procedência } & \multicolumn{6}{|c|}{ Local de Atendimento } \\
\hline & \multicolumn{2}{|c|}{ Munic. Rib. Preto } & \multicolumn{2}{|c|}{ Demais Munic. } & \multicolumn{2}{|c|}{ Total } \\
\hline & $\mathrm{N}$ & $\%$ & $\mathrm{~N}$ & $\%$ & $\mathrm{~N}$ & $\%$ \\
\hline Munic. Rib. Preto & 46.742 & 65,6 & 250 & 0,4 & 46.992 & 37,2 \\
\hline $\begin{array}{l}\text { Região estudada } \\
\text { (exceto o Munic. RP) }\end{array}$ & 18.998 & 26,7 & 54.499 & 99,1 & 73.497 & 58,2 \\
\hline Outras & 5.531 & 7,8 & 277 & 0,5 & 5.808 & 4,6 \\
\hline Total & 71.271 & 100,0 & 55.026 & 100,0 & 126.297 & 100,0 \\
\hline
\end{tabular}

cípio de Ribeirão Preto, 65,6\% eram de pacientes procedentes do próprio município, $26,7 \%$ residiam em municípios pertencentes à região estudada e $7,8 \%$ eram pacientes residentes fora da mesma. Por outro lado, dos 55.026 egressos ocorridos nos 16 hospitais dos demais municípios da região, $99,1 \%$ eram procedentes desses mesmos minicípios, sendo que $0,4 \%$ residiam no Município de Ribeirão Preto e $0,5 \%$ eram de fora da região estudada. Enquanto 18.998 pacientes dos municípios da Região procuraram leitos hospitalares do $\mathrm{Mu}$ nicípio de Ribeirão Preto, apenas 250 pacientes deste município socorreram-se dos demais leitos da região, para internação.

O Município de Ribeirão Preto parece auto-suficiente em termos de recursos para a saúde quando comparado com a região estudada, como grande centro urbano, com maiores recursos de assistência médico-hospitalar, funciona como pólo de atração para os atendimentos dos demais municípios da Região e fora dela.

É conveniente mencionar que, por outro lado, os dados disponíveis no presente trabalho não permitem avaliar a evasão de pacientes do Município de Ribeirão Preto e da Região, em direção a outros centros importantes como a Cidade de São Paulo.

A Tabela 4 apresenta a comparaçāo entre os egressos hospitalares do Município de Ribeirão Preto, obtidos por Yazlle Rocha ${ }^{18}$, em 1972, e Fávero ${ }^{6}$, em 1975, e no presente estudo em 1988. Observou-se que o volume de atendimentos feito nos hospitais do Município de Ribeirão Preto aumentou em números absolutos, de $40.832 \mathrm{em} 1972$ para $71.272 \mathrm{em}$ 1988. Proporcionalmente, houve uma diminuição dos pacientes procedentes do próprio município, de 69,1\% em 1972 para 65,6\% em 1988 , correspondendo a aumento de pacientes vindos da região estudada e de outros municípios.

O Município de Ribeirão Preto, ao longo de período contínuo, acentuou sua característica como pólo de concentração de doentes da regiāo. Esta seria uma situação desejável se os pacientes procurassem assistência em Ribeirão Preto quando fossem portadores de doenças graves que demandassem serviços especializados. Caso contrário, os dados da Tabela 4 podem sugerir que a regionalização da assistên. cia ao paciente não atingiu completamente seus objetivos.

O fluxo de pacientes entre uma área e outra é um exemplo de que a distância não é um obstáculo para o homem, quando se trata da busca de assistência. Daí que conceito de regionalização, exceto quando subordinado a rígidas imposições governamentais, e mesmo

Tabela 4 - Número e percentagem de egressos hospitalares do Município de Ribeirão Preto, segundo procedência 1970 - 1988 .

\begin{tabular}{|c|c|c|c|c|c|c|}
\hline \multicolumn{7}{|l|}{ Procedência } \\
\hline & \multicolumn{2}{|c|}{$1972^{*}$} & \multicolumn{2}{|c|}{$1970.1974^{\star *}$} & \multicolumn{2}{|c|}{1988} \\
\hline & $\mathbf{N}$ & $\%$ & $\mathrm{~N}$ & $\%$ & $\mathbf{N}$ & $\%$ \\
\hline Munic. Rib. Preto & 28.234 & 69,1 & 126.110 & 67,0 & 46.742 & 65,6 \\
\hline $\begin{array}{l}\text { Região estudada } \\
\text { (exceto o Munic. RP) }\end{array}$ & 9.755 & 23,9 & 47.856 & 25,4 & 18.998 & 26,6 \\
\hline Outras & 2.843 & 7,0 & 14.369 & 7,6 & 5.531 & 7,8 \\
\hline Total & 40.832 & 100,0 & 188.335 & 100,0 & 71.272 & 100,0 \\
\hline
\end{tabular}

- Yazlle Rocha ${ }^{18}$ (1974)

* Fávero ${ }^{8}$ (1975) 
Tabela 5 - Número e percentagem de egressos hospitalares segundo fonte de financiamento e local de atendimento.

\begin{tabular}{lcrrrrr}
\hline Fonte de & \multicolumn{7}{c}{ Local de Atendimento } \\
\cline { 2 - 7 } Financiamento & \multicolumn{2}{c}{ Munic. Rib. Preto } & \multicolumn{2}{c}{ Demais Munic. } & \multicolumn{1}{c}{ Total } \\
& $\mathrm{N}$ & $\%$ & $\mathrm{~N}$ & $\%$ & $\mathrm{~N}$ & $\%$ \\
\hline INAMPS* & 29.851 & 41,9 & 24.540 & 44,6 & 54.393 & 43,1 \\
Outras & 14.975 & 21,0 & 24.918 & 45,3 & 39.893 & 31,6 \\
Não Pagantes & 20.954 & 29,4 & 3.170 & 5,8 & 24.124 & 19,1 \\
Particular & 4.517 & 6,3 & 1.487 & 2,7 & 6.004 & 4,7 \\
SASSOM** & 428 & 0,6 & 68 & 0,1 & 496 & 0,4 \\
3 BP*** & 12 & 0,0 & $\ldots$ & $\ldots$ & 12 & 0,0 \\
Prejudicados & 534 & 0,8 & 841 & 1,5 & 1.375 & 1,1 \\
\hline Total & 71.272 & 100,0 & 55.025 & 100,0 & 126.297 & 100,0 \\
\hline
\end{tabular}

* Instituto Nacional Assistência Médica e Previdência Social

* Assistência e Seguro Social Municipal de Rib. Preto

*** Centro Social Regional do $3^{2}$ Batalhão de Polícia

assim, com ressalvas, restringe-se a um conceito teórico. Segundo Donabedian e Axel$\operatorname{rod}^{2}$, "na sua forma pura, a regionalização hospitalar visa ao planejamento unificado de um sistema de hospitais funcionalmente diferenciados e cuidadosamente coordenados, servindo em toda a sua extensão a uma região geográfica demarcada, não pelas acanhadas fronteiras políticas, mas de acordo com o modelo estabelecido, de procura e previsão de serviços médicos, de forma análogas às áreas de comércio".

As internações de pacientes na região de Ribeirão Preto são apresentadas na Tabela 5, segundo a fonte de financiamento da hospitalização. A maioria das internações ocorreu por conta do Instituto Nacional de Assistência Médica e da Previdência Social (INAMPS), $43,1 \%$, vindo a seguir a categoria "outros", $(31,6 \%)$. Nesta categoria estão incluídas as Empresas particulares e Cooperativas, como UNIMED, GOLDEN-CROSS, etc.

Em terceiro lugar, aparecem os indigentes não pagantes $(19,10 \%)$ e ocupando o quarto lugar em importância estão os particulares com $(4,7 \%)$; As demais formas de financiamento das internações como os Beneficiários de Assistência e Seguro Social do Município de Ribeirão Preto - SASSOM- e do Centro Social Regional do $3^{\circ}$ Batalhão de Polícia ( $3^{\circ}$ BP) foram inexpressivos com $0,3 \%$ e $0,1 \%$ respectivamente.

Ao cotejar os resultados das fontes de financiamento do Município de Ribeirão Preto e os da região exceto o Município, verifica-se que $6,3 \%$ dos pacientes atendidos no Municipio Ribeirão Preto são particulares e $29,4 \%$ são não pagantes, enquanto que apenas $2,7 \%$ dos atendidos nos demais municípios da $\mathrm{Re}-$ gião são particulares e 5,8\% são não pagantes.

Por outro lado, 44,6\% dos atendimentos feitos nos hospitais da regiāo são financiados pelo INAMPS enquanto que no Município de Ribeirão Preto esta percentagem é um pouco menor $(41,9 \%)$. Essas variações devem ter sua origem nas características diferenciadas dos municípios quanto à organização, disponibilidade e consumo de serviços de saúde, influenciados pelo desenvolvimento socioeconômico de cada um deles. Em nenhum dos hospitais os atendimentos foram financiados por única fonte. Dos 25 hospitais estudados, 8 são de finalidade privada-lucrativa. Era de se esperar que pelo menos estes tivessem no máximo duas fontes de financiamento: particular e "outros" que inclui convênios.

Em hipótese alguma deveriam aparecer nestes 8 hospitais internaçōes de não-pagantes. No entanto, paradoxalmente aos nāo pagantes e os financiados pela Previdência aparecem em todos os hospitais independentemente de suas finalidades.

Ao comparar os resultados da presente pesquisa, com os de outros autores, Yazlle ${ }^{18} \mathrm{e}$ Fávero $^{6}$, observou-se qual foi a tendência e as mudanças nas fontes de financiamento da assistência médico-hospitalar no Município de Ribeirão Preto, de 1970 a 1988 (Tabela 6). O INAMPS, embora continue sendo o principal responsável pelo financiamento dos atendimentos, apresentou diminuição passando de 55 a $57 \%$, na década de 70 , para $41,88 \%$, em 1988. Chama a atenção este achado, pois a tendência esperada seria de aumento, baseada 
Tabela 6 - Número e percentagem de egressos hospitalares do Município de Ribeirāo Preto segundo fonte de financiamento 1970 - 1988.

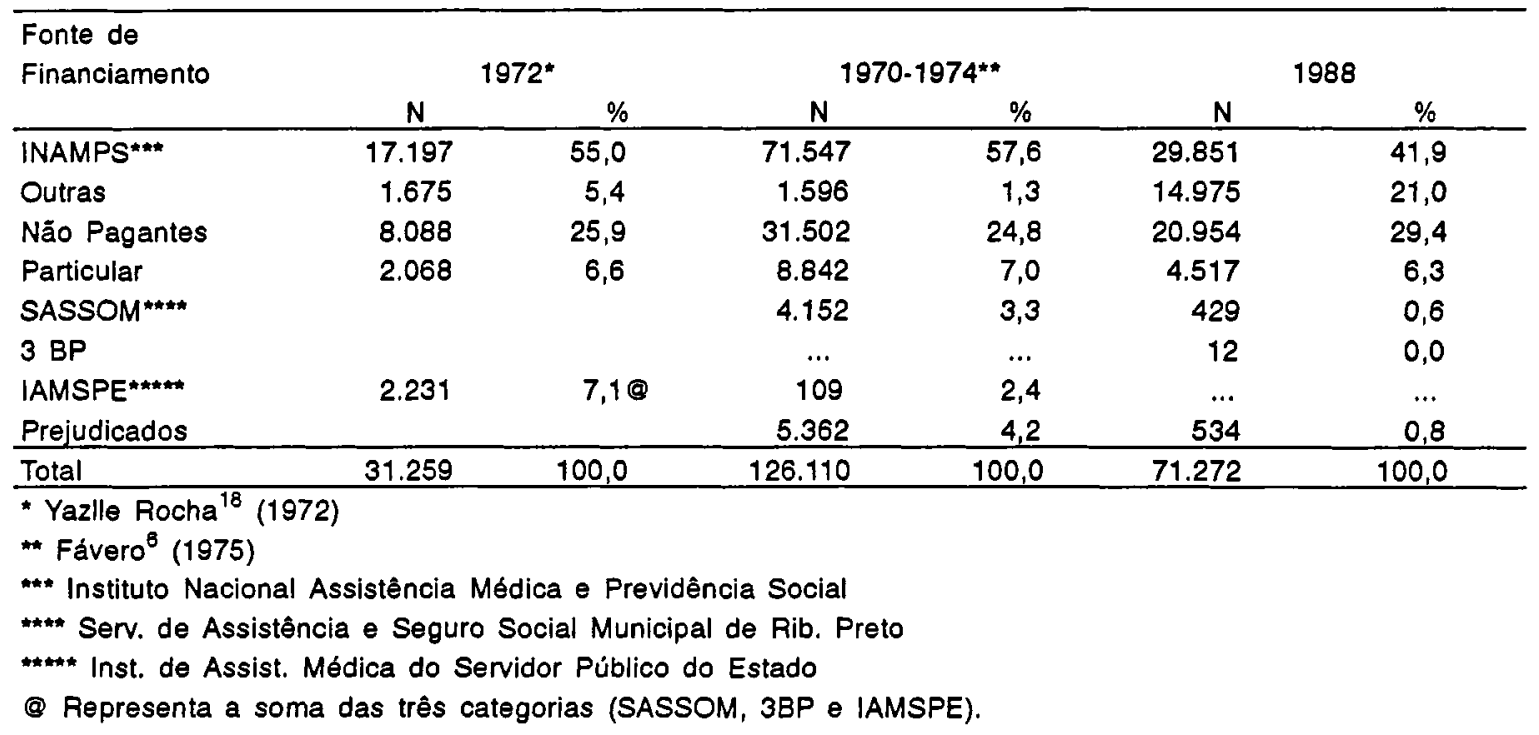

nas medidas adotadas pelo Ministério da Previdência e Assistência Social para aumentar a cobertura da população para este sistema (Ações Integradas de Saúde, Institucionalização do Sistema Unificado e Descentralizado de Saúde, entre outras).

Vê-se na Tabela 6 , que a proporção de atendimentos particulares também caiu de $6,6 \%$ para $6,4 \%$ no período. O INAMSP que contribuía com $7,1 \%$ em 1972, não participou em 1988. Chama entretanto a atenção a categoria "outros" que aumentou de $5,4 \%$, em 1972, para 21,0\%, em 1988.

Nesta categoria estão incluídas a UNIMED, GOLDEN-CROSS e outras empresas particulares de seguro saúde. Tem-se a impressão de que o aumento dos custos da assistência médico-hospitalar e atuação insatisfatória de institutos oficiais como o INAMPS têm incentivado as pessoas a procurarem, no seguro saúde privado, a desejada garantia contra riscos de elevadas despesas na eventualidade de agravo à saúde como salienta Guimarães ${ }^{8}$.

Por outro lado, este fenômeno deve estar ligado ao aumento do poder aquisitivo da população da Região considerada hoje uma das maiores rendas per capita do país, tornando-se atrativa para empresas particulares de financiamento da assistência médica, e falta de confiança, cada vez maior, no sistema previdenciário nacional.

O grupo dos não pagantes passou de $25,9 \%$ no ínicio da década de 70 para apenas $29,4 \%$; mostrando que embora tenha havido um grande desenvolvimento socioeconômico na região, o modelo capitalista de produção não foi suficiente para diminuir a parcela da população que não tem acesso à distribuição da renda.

\section{Considerações Finais}

Do ponto de vista das aplicações para o planejamento da assistência médico-hospitalar, no contexto das atuais "mudanças" na política de saúde, onde o hospital há de assumir um papel de liderança técnica e administrativa, os resultados do presente estudo se aplicam principalmente à região de Ribeirão Preto, que oferece as melhores condições para estudar a utilização dos recursos disponiveis para assistência individual, analisando os fatores sociodemográficos relacionados com essa demanda. Não será possível, no entanto, extrapolar estes resultados diretamente a qualquer cidade ou região do país, sem observar as restrições e peculiaridades de cada local.

A abordagem epidemiológica da administração dos serviços de saúde requer mais do que uma simples contabilidade dos serviços oferecidos. Uma análise epidemilógica dessa utilização e da evidência de satisfação da demanda de hospitalização deve ser relacionada à população em risco de precisar dos serviços, analisando scmpre os possiveis fatores que permeam a relação oferta/demanda.

Os resultados mostram que a distribuição 
da demanda segundo sexo e idade é semelhante à conhecida na literatura.

A regionalização da assistência é fortemente influenciada pela presença de grandes centros urbanos, e pelo desenvolvimento socioeconômico.

Há um incremento acentuado do seguro saúde privado acompanhado de uma diminuição da participação dos órgãos oficiais como modalidade de financiamento.

Há um elevado coeficiente de internação aliado à baixa utilização de leitos hospitalares.

\section{Agradecimento}

Ao Centro de Processamento de Dados Hospitalares (CPDH) do Departamento de Medicina Social da Faculdade de Medicina de Ribeirão Preto da USP, por permitir o acesso e utilização das informações cadastradas.

MONTERO D'OLEO, R. de J. \& FÁVERO, M. [The social-demographic profile of the population seeking health services in a region of the State of S.Paulo, Brazil, 1988]. Rev. Saúde públ., S. Paulo, 26: $256-63,1992$. A descriptive, retrospective study of the socio-economic profile of the population seeking health services in a Northeastern region of the State of S.Paulo was carried out and the results compared with those of previous studies. A total of 126,297 patients discharged from 25 general hospitals of the region in 1988 were studied according to sex, age group, residence, source of financing and hospital indicators. The results of this study show that the distribution of the demand according to sex and age is similar to that known from the relevant literature. These results suggest that the regionalization of assistance, in spite of planning and of pertinent legislation, is unsatisfactory, strongly influenced by the presence of large urban centers and socio-economic development. Various sources of financing are used together with a considerable increase of private health insurance and a diminishing participation of governmental institutions over the years. The region studied presents a high rate hospitalization as well as rate of hospital bed utilization.

Keywords: Health services research. Hospital, general, statistics. Health services needs and demand, trends. Socio-economic factors.

\section{Referências Bibliográficas}

1. BARROS, M.B. de A. Estudo de morbidade hospitalar no Município de Ribeirão Preto, em 1975. Ribeirão Preto, 1977. [Dissertação de Mestrado - Faculdade de Medicina de Ribeirāo Preto da USP].

2. DONABEDIAN, A. \& AXELROD, S.J. Organizing medical care programs to meet health needs. Ann.Amer.Acad.Pol.soc.sci., 337: 45-56, 1961.
3. DONABEDIAN, A. Aspects of medical care administration; specifying requirements for health care. Cambridge, Mass., Harvard University Press, 1973.

4. DEVER, G.E. Epidemiology in health services management. Rockville, Med. Aspen Publ., 1984.

5. FAVERO, M. et al. Organização de um centro de informática hospitalar em nivel local. Rev. paul. Hosp., 21: 151-7, 1973.

6. FÁVERO, M. Estudo da duração da intemação em hospitais gerais de Ribeirão Preto. Ribeirão Preto, 1975. [Tese de Livre-Docência - Faculdade de Medicina de Ribeirão Preto da USP].

7. GRAVES, E.J. Utilization of short-stay by diagnosis: United States, 1986. Vital Hlth Stat. Ser. 13(96), 1987.

8. GUIMARÃES, C. Leitos de assistência hospitalar geral do Vale do Paraiba. São Paulo, 1979. [Tese de Livre-Docência - Faculdade de Saúde Pública da USP].

9. LEBRÃO, M.L Morbidade hospitalar no Vale do Paraiba, 1975. São Paulo, 1982. [Tese de Doutorado - Faculdade de Saúde Pública da USP].

10. NAVARRO, V. Planning for the distribution of personal health services. Publ. Hlth Rep., 84: 573-8, 1969.

11. NEWELL, D.J. Problems of stimating the demand for hospital bed. J.chron.Dis., 17: 749-53, 1964.

12. ORGANIZACION PANAMERICANA DE LA SALUD. Atención médica: bases para la formación de una politica continental. Washington, DC, 1962. (Publicacion Científica, 70).

13. ORGANIZACION PANAMERICANA DE LA SALUD. Las condições de salud de las Américas; informe final. Washington, DC, 1970. (Publicacion Cientifica, 207).

14. ORGANIZACION PANAMERICANA DE LA SALUD. Plan decenal de salud para las Américas: informe final. Washington, DC, 1973. (Documento Oficial, 118).

15. ORGANIZACION PANAMERICANA DE LA SALUD. Las condiciones de salud en las Américas: 1981-1984. Washington, DC, 1986. (Publicacion Cientifica, 500).

16. POKR AS, R. et al. Trends in hospital utilization: United States, 1965-86. Vital Hlth Statist. Ser. 13 (105), 1989.

17. SINGER, P. et al. Prevenir e curar: o controle social através dos serviços de saúde. Rio de Janeiro, Forense Universitária, 1979.

18. YAZLLE ROCHA, J.S. Estudo da utilização de leitos hospitalar do Município no ano de 1972. Ribeirão Preto, 1974. [Tese de Doutorado - Faculdade de Medicina de Ribeiräo Preto da USPJ.

19. YAZLLE ROCHA, J.S. Assistência médico-hospitalar, Ribeirão Preto (SP), 1972-1976: condições para uma política de assistência hospitalar. Saúde Deb., (6): 38-41, 1978

20. YAZLLE ROCHA, J.S. et al. Padrōes de morbidade hospitalar considerações para uma política de assistência médico - hospitalar. Rev. Saúde públ., S.Paulo, 14: 58-64, 1980. 